\title{
Ein Satz über vertauschbare Matricen und seine Anwendung in der Theorie linearer Differential- gleichungen.
}

Von J. Plemelj in Wien.

Die particulären Integrale, welche ein Fundamentalsystem einer linearen Differentialgleichung bilden, erleiden bei gemeinsamer Fortsetzung auf einem Wege, der die Coefficienten der Differentialgleichung in die ursprünglichen Werte zurückführt, bekanntlich eine lineare Substitution (Fundamentalsubstitution) und jede mit dieser ähnliche Substitution gehört als Fundamentalsubstitution zu einem bestimmten Fundamentalsysteme. Von grundlegender Bedeutung für die Theorie linearer Differentialgleichungen ist der Satz, dass sich zu jeder linearen Substitution eine andere ähnliche von weit einfacherer (canonischer) Gestalt auffinden lässt, welche als Fundamentalsubstitution aufgefasst - nach Riemann und Fuchs ${ }^{1}$ ) - mit Leichtigkeit die analytische Form der Integrale im Bereiche der Fortsetzung erkennen lässt.

Es gehört aber im allgemeinen zu jedem Fortsetzungswege, von der angegebenen Beschaffenheit ein eigenes Fundamentalsystem, Fortsetzungswegen jedoch, denen vertauschbare Fundamentalsubstitutionen zukommen, entspricht ein gemeinsames canonisches Fundamentalsystem. Die Folge dieses Satzes ist, dass die Integration einer linearen Differentialgleichung, deren Monodromiegruppe aus lauter vertauschbaren Substitutionen besteht, auf eine Kette linearer Differentialgleichungen erster Ordnung mit eindeutigen, bei Differentialgleichungen der Fuchs'schen Classe in einer mehrblättrigen Riemann'schen Fläche auch rationalen Coefficienten, zurückführbar ist.

1) Riemann, Inauguraldissertation, Göttingen 1851.

Crelle's Journal, Band 54.

Fuchs, Crelle's Journal, Band 66.

Vgl. Schlesinger: Theorie der Differentialgleichungen, Band I. Leipzig 1895. Seite $96 \mathrm{ff}$. , wo die bezügliche Literatur angegeben ist. 
Dieses Ergebnis, das in der Algebra bei den Gleichungen, deren Galois'sche Gruppe eine Abel'sche ist, ein Analogon besitzt, folgt aus einem Satze über vertauschbare Matricen oder bilineare Formen, deren Theorie seit Weierstraß besonders durch Frobenius weitgehend ausgebildet wurde. ${ }^{1}$ )

\section{1.}

Wir bezeichnen mit $\Omega_{n}$ das Größengebiet, dass aus sämmtlichen linearen Formen von $n$ bestimmten Größen $z_{1}, z_{2}, \ldots, z_{n}$ gebildet wird. Zwischen $z_{1}, z_{2}, \ldots, z_{n}$ soll keine lineare homogene Beziehung bestehen, es lässt sich dann $\Omega_{n}$ nicht auf ein Gebiet zurückführen, in welchem sämmtliche Bestandtheile (Elemente) durch weniger als $n$ Größen linear darstellbar wären.

Wird eine durch das Coefficientensystem (Matrix)

$$
\Theta=\left(\begin{array}{l}
a_{11}, a_{21}, \ldots, a_{1 n} \\
a_{21}, a_{22}, \ldots, a_{2 n} \\
\ldots, \ldots, \ldots, \ldots \\
a_{n 1}, a_{n 2}, \ldots, a_{n n}
\end{array}\right)
$$

charakterisierte lieneare Substitution auf die Größen $z_{1}, z_{2}, \ldots, z_{n}$. ausgeübt und gehen daraus die Größen $\Theta z_{1}, \Theta z_{2}, \ldots, \Theta z_{n}$ hervor, so wollen wir dies durch das Symbol

$$
\left(\Theta z_{1}, \Theta z_{2}, \ldots, \Theta z_{n}\right)=\Theta\left(z_{1}, z_{2}, \ldots, z_{n}\right)
$$

anzeigen, wobei also durch $(2 a)$ die $n$ Gleichungen

$$
\begin{gathered}
\Theta z_{\varkappa}=a_{\varkappa 1} z_{1}+a_{\varkappa 2} z_{2}+\cdots+a_{\varkappa n} z_{n} \\
x=1, \varkappa, \ldots, n
\end{gathered}
$$

ersetzt werden. Wenn man dann irgend einem Elemente

$$
z=c_{1} z_{1}+c_{2} z_{2}+\cdots c_{n} z_{n}
$$

aus $\Omega_{n}$ das Element

$$
\Theta z=c_{1} \Theta z_{2}+c_{2} \Theta z_{2}+\cdots+c_{n} \Theta z_{n}
$$

an die Seite stellt, so ist im Gebiete $\Omega_{n}$ auf diese Weise durch die Substitution $\Theta$ eine gewisse Zuordnung der Elemente zu einander durch die Gleichungen (2) bestimmt.

Lassen sich die Zahlen $1,2,3, \ldots, n$ derart in Gruppen eintheilen, dass jede Zahl in einer und nur einer Gruppe vorkommt

1) Weierstra $\beta$, Monatsberichte der Berl. Akademie 1868.

Frobenius, Crelle's Journal. Band 84.

Sitzungsberichte der Berl. Akademie 1896.

Voss, Sitzungsberichte. der München. Akademie 1889.

Weyr Ed, Monatshefte für Mathematik und Physik. Band 1. 
und dass in (1) sämmtliche Coefficienten $a_{i k}$ verschwinden, wenn nicht dio Indices $i$ und $k$ zur selben Gruppe gehören, so sagen wir, dass die Matrix zerfällt. Sind z. B. $\alpha, \beta, \ldots, x$ und $\lambda, \mu, \ldots, \sigma$ zwei Gruppen, in welche die Zahlen $1,2, \ldots, n$ auf diese Weise sich sondern lassen, dann können wir statt $(2 a)$ die zwei Substitutionen

$$
\begin{gathered}
\left(\Theta z_{\alpha}, \Theta z_{\beta}, \ldots, \Theta z_{\gamma}\right)=\left(a_{i k}\right)\left(z_{\alpha}, z_{\beta}, \ldots, z_{*}\right) \\
i \\
k\}=\alpha, \beta, \ldots, \% \\
\left(\Theta z_{\lambda}, \Theta z_{\mu}, \ldots, \Theta z_{\sigma}\right)=\left(a_{l m}\right)\left(z_{\lambda}, z_{\mu}, \ldots, z_{\sigma}\right) \\
\left.\begin{array}{c}
l \\
m\}
\end{array}\right\}=\lambda, \mu, \ldots, \sigma
\end{gathered}
$$

schreiben und das Bestehen zweier oder mehrerer Substitutionen dieser Form ist äquivalent mit der Zerfällbarkeit der Matrix.

Durch die Elemente $z_{\alpha}, z_{\beta}, \ldots, z_{\varkappa}$ kann ein aus sümmtlichen linearen Formen bestehendes Größengebiet definiert werden, welches die Eigenschaft besitzt, dass anch die durch die Substitution $\Theta$ nach (2) zugeordneten Elemente wieder zum selben Gebiete gehören. Wir wollen es ein Theilgebiet in $\Omega_{n}$ nennen.

Setzen wir analog, wie in $(2 a)$

$$
\left(\Theta^{\lambda} z_{1}, \Theta^{\lambda} z_{2}, \ldots, \Theta^{\lambda} z_{n}\right)=\Theta^{\lambda}\left(z_{1}, z_{2}, \ldots, z_{n}\right),
$$

wo, wie üblich, $\Theta^{\lambda}$ die $\lambda$-mal hintereinander mit sich selbst componierte Substitution $\Theta$ bedeutet, so haben wir allgemein

$$
\Theta^{2} \theta^{\lambda} z=\theta^{*+\lambda} z=\theta^{2} \Theta^{x} z
$$

und speciell $\theta^{1} z=\theta z, \theta^{0} z=z$ zu nehmen.

Werden die Zuordnungen in zweifacher Weise durch zwei vertauschare Substitutionen $\Theta_{1}$ and $\Theta_{2}$ definiert, so ergibt sich aus

$$
\Theta_{1}^{z} \Theta_{2}^{2}\left(z_{1}, z_{2}, \ldots, z_{n}\right)=\Theta_{2}^{\lambda} \Theta_{1}^{*}\left(z_{1}, z_{2}, \ldots, z_{n}\right)
$$

die Gleichung

$$
\theta_{1}^{z} \theta_{2}^{\lambda} z=\theta_{2}^{\lambda} \theta_{1}^{x} z
$$

wobei die Bedeutung von $\Theta_{1}^{\alpha} \Theta_{2}^{\lambda} z$ unmittelbar klar ist, da $\Theta_{2}^{\lambda} z$ ein Element aus $\Omega_{n}$ ist.

Jede Größ̉e der Form

$$
\mathfrak{A}(z)=\sum_{\alpha_{1} a_{2}} \mathfrak{a}_{\alpha_{1} \alpha_{2}} \Theta_{1}^{\alpha_{1}} \Theta_{2}^{\alpha_{2}} z,
$$

wo die $\mathfrak{a}_{\alpha_{1} a_{2}}$ beliebige Constanten sind, gehört auch zum Gebiete $\Omega_{n}$, weshalb wir mit Riucksicht auf (4b) bekommen:

$$
\Theta_{1}^{\beta_{1}} \Theta_{2}^{\beta_{2}} \mathfrak{A}(z)=\mathscr{I}\left(\Theta_{1}^{\beta_{1}} \Theta_{2}^{\beta_{2}} z\right) \text {. }
$$


Setzt man nun $\mathfrak{A}(z)$ als $x$ in

$$
\mathfrak{B}(x)=\sum_{\beta_{1} \beta_{2}} \mathfrak{b}_{\beta_{1} \beta_{2}} \Theta_{1}^{\beta_{1}} \Theta_{2}^{\beta_{2}} x
$$

ein und schreibt dafür $\mathfrak{B} \mathfrak{P}(z)$, so folgt

oder nach (6)

$$
\mathfrak{B} \mathfrak{A}(z)=\sum_{\beta_{1} \beta_{2}} \mathfrak{G}_{\beta_{1} \beta_{2}} \Theta_{1}^{\beta_{1}} \Theta_{2}^{\beta_{2}} \mathfrak{A}(z)
$$

$$
\mathfrak{B} \mathfrak{Z}(\boldsymbol{z})=\mathfrak{A} \mathfrak{B}(\mathfrak{a})
$$

und man sieht zugleich, dass $\mathfrak{M} \mathfrak{B}(z)$ ebenso zu bilden ist, als wären $\mathfrak{A}$ und $\mathfrak{B}$ die algebraischen Ausdrücke

$$
\mathfrak{A}=\sum_{a_{1} \alpha_{2}} \mathfrak{a}_{\alpha_{1} \alpha_{2}} \Theta_{1}^{a_{1}} \Theta_{2}^{a_{2}}, \mathfrak{B}=\sum_{\beta_{2} \beta_{2}} \mathfrak{H}_{\beta_{1} \beta_{2}} \Theta_{1}^{\beta_{1}} \Theta_{2}^{\beta_{3}}
$$

von $\Theta_{1}$ und $\theta_{2}$ und $\mathfrak{A} \mathfrak{B}$ ihr Product, $\approx$ aber nur ein anzuhängender Factor. Mit Gröben der Form (5) wird man also ebenso zu rechnen haben, wie mit algebraischen Ausdrücken von $\theta_{1}$ und $\theta_{2}$, man wird in diesem Sinne von ihren Producten und Theilern sprechen können. Wir lassen sie durch gothische Zeichen hervortreten.

Werden durch eine lineare Substitution

$$
\left(x_{1}, x_{2}, \ldots, x_{n}\right)=\mathrm{T}\left(z_{1}, z_{2}, \ldots, z_{n}\right)
$$

$n$ Größen $x_{1}, x_{2}, \ldots, x_{n}$ bestimmt und versehwindet nicht die Determinante der Matrix $\mathrm{T}$, so lassen sich die durch (8a) repräsentierten $n$ Gleichungen nach $z_{1}, z_{2}, \ldots, z_{n}$ auflösen, und man bekommt in üblicher Bezeichnungsweise

$$
\left(z_{1}, z_{2}, \ldots, z_{n}\right)=\mathrm{T}^{-1}\left(x_{1}, x_{2}, \ldots, x_{n}\right) .
$$

Sämmtliche Elemente aus $\Omega_{n}$ sind also auch als Linearformen von $x_{1}, x_{2}, \ldots, x_{n}$ darstellbar. Zwischen $\Theta x_{1}, \Theta x_{2}, \ldots, \Theta x_{n}$ und $x_{1}, x_{2}, \ldots, x_{n}$ besteht nach (8) und (2) die Beziehung

$$
\left(\Theta x_{1}, \Theta x_{2}, \ldots, \Theta x_{n}\right)=\mathrm{T} \Theta \mathrm{T}^{-1}\left(x_{1}, x_{2}, \ldots, x_{n}\right)
$$

und es wird, wenn

$$
\begin{aligned}
x & =c_{1} \cdot x_{1}+c_{2} \cdot x_{2}+\cdots+c_{n} \cdot x_{n} \\
\Theta x & =c_{1} \Theta x_{1}+c_{2} \Theta x_{2}+\cdots+c_{n} \Theta x_{n}
\end{aligned}
$$

gesetzt wird, dieselbe Zuordnung der Elemente in $\Omega_{n}$ wie durch die Gleichungen (2) bestimmt. Jedes so bestimmte System ron 
$n$ linearen unabhängigen Elementen wollen wir ein $\mathrm{Fu}$ udamentalsystem von $\Omega_{n}$ nennen.

Wir wollen zunächst zu einer vorgegebenen Substitution $\Theta$ eine solche Substituton $T$ aufsuchen, dass dadurch das Zerfallen von $\mathrm{T} \Theta \mathrm{T}^{-1}$ bewirkt wird. Das Resultat dieser Ableitung wird dann anf vertauschbare Substitutionen angewendet.

2.

Es soll zwischen $\Theta x_{1}, \Theta x_{2}, \ldots, \Theta x_{\kappa}$ und $x_{1}, x_{2}, \ldots, x_{*}$ die lineare Substitution

$$
\left(\Theta x_{1}, \Theta x_{2}, \ldots, \Theta x_{r}\right)=S\left(x_{1}, x_{2}, \ldots, x_{x_{2}}\right)
$$

mit dem Coefficientensystem

$$
S=\left(\begin{array}{c}
\alpha_{11}, \alpha_{12}, \ldots, \alpha_{1 \varkappa} \\
\alpha_{21}, \alpha_{22}, \ldots, \alpha_{2 \varkappa} \\
\ldots, \ldots, \ldots, \ldots \\
\alpha_{x 1}, \alpha_{r 2}, \ldots, \alpha_{z \varkappa}
\end{array}\right)
$$

bestehen; durch $x_{1}, x_{2}, \ldots, x_{x}$ wird dann in $\Omega_{n}$ ein Theilgebiet $\Omega_{x}$ abgegrenzt, in welchem höchstens je $*$ Elemente linear unabhängig sind. Gehört also $x$ zu $\Omega_{x}$, so muss es zwischen den $x+1$ Elementen

$$
x, \theta x, \theta^{2} x, \ldots, \theta^{*} x
$$

eine lineare Beziehung

$$
\Theta^{x} x+a_{1} \Theta^{x-1} x+\cdots+a_{x-1} \Theta x+a_{x} x=0
$$

geben, in welcher $a_{1}, a_{2}, \ldots, a_{\varkappa}$ bestimmte Constanten sind. Die Elemente $x, \Theta x, \ldots, \Theta^{x-1} x, \Theta^{x} x$ sind sämmtlich durch $x_{1}, x_{2}, \ldots, x_{x}$ linear darstellbar. Wir setzen

$$
\begin{gathered}
\Theta^{\lambda} x=c_{1}^{\lambda} x_{1}+c_{2}^{\lambda} x_{2}+\cdots+c_{x}^{\lambda} x_{x} \\
\lambda=0,1, \ldots, k-1, x .
\end{gathered}
$$

Um (11) zo erfüllen, ist es nothwendig und hinreichend, dass die Gleichungen

$$
\begin{aligned}
& 0=c_{1}^{*}+a_{1} c_{1}^{n-1}+\cdots+a_{\varkappa-1} c_{1}^{1}+a_{\varkappa} c_{1}^{0} \\
& 0=c_{2}^{x}+a_{1} c_{2}^{n-1}+\cdots+a_{x-1} c_{2}^{1}+a_{n} c_{2}^{0} \\
& 0=c_{x}^{*}+a_{1} c_{x}^{n-1}+\cdots+a_{x-1} c_{x}^{1}+a_{n} c_{x}^{0}
\end{aligned}
$$


durch $a_{1}, a_{2}, \ldots, a_{*}$ erfüllt werden. Fügt man noch

$$
\Re=\Theta^{x}+a_{1} \Theta^{n-1}+\cdots+a_{n-1} \Theta+a_{\varkappa}
$$

hinzu $\Omega$ als algebraischen Ausdruck ron $\Theta$ auffassend, so folgt durch Elimination von $a_{1}, a_{2}, \ldots, a_{\varkappa}$ aus (11) und (14)

$$
D . \Re=\left|\begin{array}{c}
\Theta^{\alpha}, \Theta^{*-1}, \ldots, \Theta, 1 \\
c_{1}^{*}, c_{1}^{\alpha-1}, \ldots, c_{1}^{1}, c_{1}^{0} \\
c_{2}^{*}, c_{2}^{*-1}, \ldots, c_{2}^{1}, c_{2}^{0} \\
\ldots, \ldots \ldots \ldots, \ldots, \ldots \\
c_{n}^{*}, c_{n}^{*-1}, \ldots, c_{n}^{1}, c_{n}^{0}
\end{array}\right|,
$$

wo die Subdeterminante von $\theta^{*}$ mit $D$ bezeichnet wurde. Durch Subtraction jeder $\Theta$-fachen Spalte von der vorangehenden findet man

$$
\begin{gathered}
D . \Re=\left(\begin{array}{c}
-1)^{x}\left|c_{v}^{\lambda}-\Theta . c_{\nu}^{\lambda-1}\right| \\
\lambda \\
\nu
\end{array}\right\}=1,2, \ldots, x,
\end{gathered}
$$

Bedenkt man, dass $\Theta^{\lambda} x=\theta \Theta^{\lambda-1} x$, so erhält man aus (12) und (10) zur Bestimmung der $c_{v}^{\lambda}$ durch die Coefficienten $c_{1}^{0}, c_{2}^{0}, \ldots, c_{x}^{0}$ die Recursionsformel

$$
c_{v}^{\lambda}=c_{1}^{\lambda-1} \alpha_{1 \nu}+c_{2}^{\lambda-1} \alpha_{2 \nu}+\cdots+c_{z}^{\lambda-1} \alpha_{x \nu} .
$$

Mit Hilfe dieser Formel zerfällt die Determinate $\left|c_{\nu}^{\lambda}\right|$ sofort in

$$
\begin{aligned}
& \left|c_{p}^{\lambda}\right|=D \quad\left|a_{\lambda v}\right| \\
& \left.\begin{array}{l}
\lambda \\
v
\end{array}\right\}=1,2, \ldots, x \text {. }
\end{aligned}
$$

In (15) wird $\theta . c_{v}^{\lambda-1}$ von $c_{v}^{\lambda}$ subtrabiert, indem man rechts nur $\alpha_{v v}$ um $\Theta$ vermindert, weshalb ähnlich wie $\left|c_{v}^{\lambda}\right|$ auch die Determinante $\left|c_{v}^{\lambda}-\Theta c_{\nu}^{\lambda-1}\right|$, wie folgt

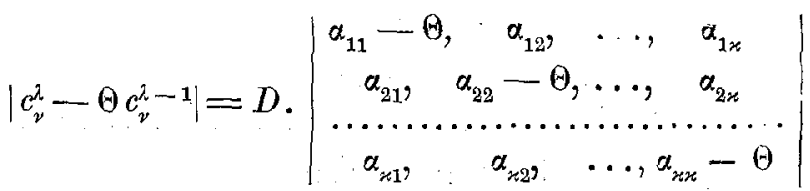

$$
\begin{aligned}
& \left.\begin{array}{l}
\lambda \\
v
\end{array}\right\}=1,2, \ldots, x
\end{aligned}
$$

gespalten werden kann. Demnach hat man aus

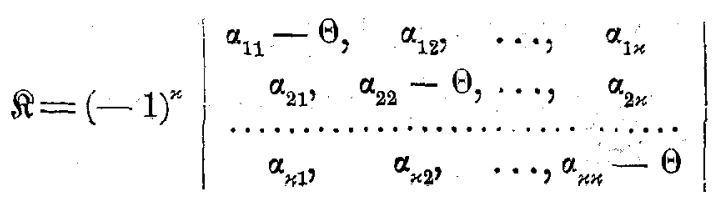


den algebraischen Ausdruck

$$
\widehat{\Re}=\Theta^{*}+a_{1} \Theta^{r-1}+\cdots+a_{r-1} \Theta+a_{r}
$$

zu bestimmen, es werschwindet dann nach (11) ^und (14), weil die Coefficienten $c_{1}^{0}, c_{2}^{0}, \ldots, c_{*}^{0}$ ausgefallen sind,

$$
\mathfrak{K}(z)=\theta^{*} z+a_{1} \Theta^{*-1} z+\ldots+a_{x-1} \Theta \approx+a_{x} z
$$

für jedes Element aus $Q_{\varkappa} . \hat{\Re}(z)$ nennen wir den zur Substitution $S=\left(\alpha_{\lambda_{\mu}}\right)$ (zum Gebiete $\left.\Omega_{\kappa}\right)$ gehörigen charakteristischen A usdruck.

Die Coefficienten $a_{1}, a_{2}, \ldots, a_{x}$, die sich so ergeben haben, sind sämmtlich endlich und befriedigen das Gleichungssystem (13) identisch, weshalb es nicht nothwendig ist den Fall, wo bei beliebiger Wahl von $c_{1}^{0}, c_{2}^{0}, \ldots c_{x}^{0}$ die Determinante $D$ verschwindet, gesondert zu betrachten. Es kann aber $\mathfrak{A}(z)$ auch von der Wahl des Fundamentalsystems in $\Omega_{x}$ individuell gar nicht abhängen, $\mathrm{d} . \mathrm{h}$. man muss durch dieses Verfahren aus jeder Substitution $A S A^{-1}$ in $\Omega_{*}$ denselben Ausdruck $\mathfrak{K}(z)$ bekommen. Dies lässt sich folgendermaßen einsehen:

Könnten sich nämlich zwei verschiedene Ausdrücke $\mathfrak{A}(z)$ und $\Re_{1}(z)$ ergeben, so wäre $\Re(z)-\Re_{1}(z)=0$ eine Relation, welche von sämmtlichen Elementen aus $\Omega_{\varkappa}$ erfüllt wäre, zudem aber einen niedrigeren als den $x$-ten Grad hätte. Wenn wir nun über die $\alpha_{\lambda \mu}$ keine Voraussetzung machen, kann es eine solche Relation nicht geben, denn das zu (13) analoge Gleichungssystem enthielte sonst weniger zu bestimmende Größen als Gleichungen. Diese würden aber im allgemeinen keine Lösung zulassen. Es ist also $\Re_{1}(z)=\Re(z)$ eine identische Gleichbeit, welche folglich auch durch Specialfälle nicht gestört werden kann.

Bestimmt man aus

die Ausdrücke

$$
\mathfrak{F}=(-1)^{n}\left|\begin{array}{cccc}
a_{11}-\Theta, & a_{12}, & \ldots & a_{1 n} \\
a_{21}, & a_{22}-\Theta, & \ldots & a_{2 n} \\
\ldots \ldots \ldots \ldots \ldots \ldots \ldots \ldots \ldots & \ldots \ldots \ldots \\
a_{n 1}, & a_{n 2}, & \ldots & a_{n n}-\Theta
\end{array}\right|
$$

$$
\begin{aligned}
& \mathfrak{F}=\Theta^{n}+a_{1} \theta^{n-1}+\ldots+a_{n-1} \Theta+a_{n} \\
& \mathfrak{F}(z)=\Theta^{n} z+a_{1} \theta^{n-1} z+\ldots+a_{n-1} \Theta z+a_{n} z,
\end{aligned}
$$

so verschwindet $\mathfrak{F}(z)$ für jedes Element aus $\boldsymbol{Q}_{n}$. Man nennt $\mathfrak{F}=\mathbf{0}$ die zur Substitution $\Theta$ gehörige charakteristische G leichung 
3.

Es sei $\mathfrak{M}(z)$ der charakteristische Ausdruck $\mu$-ten Grades in einem Gebiete $\Omega_{\mu}$, in welchen sämmtliche Bestandtheile durch die $\mu$ linear unabhängigen Elemente $z_{1}, z_{2}, \ldots z_{\mu}$ linear darstellbar sind.

Zerlegen wir $\mathfrak{M}(z)$ in

$$
\mathfrak{M}(z)=\mathfrak{A} \mathfrak{B}(z),
$$

so können wir unter der Voraussetzung, dass $\mathfrak{A}(z)$ und $\mathfrak{B}(z)$ theilerfremd sind, zwei derartige Ausdrücke $\mathfrak{A}_{1}(z)$ und $\mathfrak{B}_{1}(z)$ nach dem Algorithmus zur Bestimmung des größten gemeinsamen Theilers ermitteln, dass

$$
z=\mathfrak{A}_{1} \mathfrak{B}(z)+\mathfrak{B}_{1} \mathfrak{A}(z) .
$$

Wir setzen nun

$$
\begin{aligned}
& x=\mathfrak{A}_{1} \mathfrak{B}(\approx), \\
& y=\mathfrak{B}_{1} \mathfrak{A}(z)
\end{aligned}
$$

und haben für die Elemente $x$ und $y$ die Gleichungen

$$
\begin{aligned}
& \mathfrak{A}(x)=0, \\
& \mathfrak{B}(y)=0 .
\end{aligned}
$$

Wenn wir auf diese Weise das Fundamentalsystem $z_{1}, z_{2}, \ldots z_{\mu}$ aus $\Omega_{\mu}$ zerlegen, so bekommen wir zwei Systeme

$$
\begin{aligned}
& x_{1}, x_{2}, \ldots x_{\mu} \\
& y_{1}, y_{2}, \ldots y_{\mu},
\end{aligned}
$$

deren Elemente bezüglich den Gleichungen $\mathfrak{A}(z)=0$ und $\mathfrak{B}(z)=0$ genügen, und durch welche sich zufolge (18) jedes Element in $\Omega_{\mu}$ linear ausdrücken lässt. Da aber umgekehrt jedes dieser Elemente (20) auch durch $z_{1}, z_{2}, \ldots z_{\mu}$ ausdrückbar ist, so gibt es in (20) genau $\mu$ linear unabhängige Elemente, welche folglich $z_{1}, z_{2}, \ldots z_{\mu}$ ersetzen können.

Wenn nun in (20) unter den $x$

und ebensỏ

$$
x_{1}, x_{2}, \ldots x_{x}
$$

$$
y_{1}, y_{2}, \ldots y_{2}
$$

unter den $y$ die sämmtlichen von einander linear unabhängigen Elemente bedeuten, dann kann es auch keine Relation der Form

$$
a_{1} x_{1}+a_{2} x_{2}+\ldots+a_{\varkappa} x_{x}=b_{1} y_{1}+b_{2} y_{2}+\ldots+b_{\lambda} y_{\lambda}
$$

geben, denn das Element

$$
\begin{aligned}
z & =a_{1} x_{1}+a_{2} x_{2}+\ldots+a_{x} x_{x} \\
& =b_{1} y_{1}+b_{2} y_{2}+\cdots+b_{\lambda} y_{2}
\end{aligned}
$$


könnte, da es $\mathfrak{A}(z)$ und $\mathfrak{B}(z)$ zugleich zum Verschwinden bringen würde, nach (18) selbst von Null nicht verschieden sein. Es ist also $x+\lambda=\mu u$ und $(21 a)$ and $(21 b)$ bildene in Fundamentalsystem in $Q_{\mu}$.

Da aber

$$
\mathfrak{A} \Theta x=\Theta \mathfrak{A}(x)
$$

ist, so genügen die Elemente

$$
\Theta x_{1}, \Theta x_{2}, \ldots \Theta x_{x}
$$

selbst der Relation $\mathfrak{A}(z)=0$ und müssen, da die Gleichungen $\mathfrak{A}(z)=0, \mathfrak{B}(z)=0$ keine gemeinsame Lösung besitzen, durch

$$
x_{1}, x_{2}, \ldots x_{x}
$$

allein linear darstellbar sein. Diese Darstellung sei

und analog

$$
\begin{gathered}
\left(\Theta x_{1}, \Theta x_{2}, \ldots \Theta x_{\varkappa}\right)=\left(\alpha_{i k}\right)\left(x_{1}, x_{2}, \ldots x_{\varkappa}\right) \\
\left.i_{k}\right\}=1,2, \ldots, \varkappa
\end{gathered}
$$

$$
\begin{aligned}
& \left(\Theta y_{1}, \Theta y_{2}, \ldots \Theta y_{\lambda}\right)=\left(\beta_{i k}\right)\left(y_{1}, y_{2}, \ldots y_{\lambda}\right) \\
& \left.\begin{array}{l}
i \\
k
\end{array}\right\}=1,2, \ldots, \lambda
\end{aligned}
$$

Nach dem im zweiten Abschnitt entwickelten Verfahren ergibt sich aus (22a) ein Ausdruck $\mathfrak{K}(z)$ von $\boldsymbol{x}$-ten Grade, der für sämmtliche Elemente $x_{1}, x_{2}, \ldots x_{x}$ verschwindet und ebenso soll $\mathfrak{Q}(z)$ dieselbe Eigenschaft bezüglich der Elemente $y_{1}, y_{2}, \ldots y_{\lambda}$ besitzen.

Die Größen $x_{1}, x_{2}, \ldots x_{\#}, y_{1}, y_{2}, \ldots y_{\lambda}$ constituieren aber in $\Omega_{\mu}$ ein Fundamentalsystem, es ergibt sich also nach Abschnitt 2

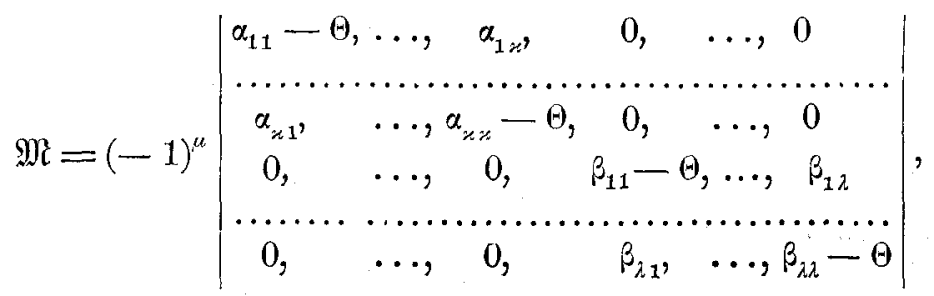

d h.. die charakteristische Function $\mathfrak{M}(z)$ zerfällt genau in

$$
\mathfrak{M}(z)=\mathfrak{K} \Omega(z) \text {. }
$$

Ist nun $\Theta z-\omega z$ ein Theiler von $\mathscr{R}(z)$, so gibt es mindestens ein nicht verschwindendes Element

für welches

$$
x=c_{1} x_{1}+c_{2} x_{2}+\ldots+c_{x} x_{2}
$$

$$
\theta x-\omega x=0
$$


wird. Die nothwendige und hinreichende Bedingung dafür bildet mit Rücksicht auf (22a) die Möglichkeit der Bestimmung eines Systems nicht sämmtlich verschwindender $c_{1}, c_{2}, \ldots c_{\text {r }}$ aus den Gleichungen

$$
\begin{aligned}
& \left(\alpha_{11}-\omega\right) c_{1}+\alpha_{21} c_{2}+\ldots+\alpha_{x 1} c_{x}=0 \\
& \alpha_{12} c_{1}+\left(\alpha_{22}-\omega\right) c_{2}+\ldots+\alpha_{x 2} c_{x}=0 \\
& \alpha_{1 x} c_{1}+\alpha_{2 x} c_{2}+\ldots+\left(\alpha_{x x}-\omega\right) c_{x}=0
\end{aligned}
$$

wozu das Verschwinden der Determinante $\Re$ in (16) für $\Theta=\omega$ erfordert wird, aber auch hinreicht. $\theta \approx-\omega z$ ist also ein Theiler von $\mathscr{R}(z)$. Theiler dieser Form wollen wir Linearth eiler nennen und mit $(5)$ bezeichnen.

Es gilt also der

Satz I. Jeder Lineartheiler der zu einem Gebiete gehörigen charakteristischen Function versehwindet mindestens für ein Element dieses Gebietes.

Hätte nun $\mathfrak{R}(z)$ mit $\mathfrak{B}(z)$ irgend einen Lineartheiler $\mathbb{E}(z)$ gemeinsam, so gäbe es nach diesem Satze ein nicht verschwindendes zu $\mathfrak{S}(z)=0$ gehöriges Element, für welches $(\xi(z)=0$ wäre, wonach also, da $(\mathbb{E}(z)$ als Theiler von $\mathfrak{B}(z)$ angenommen wurde, die beiden Gleichungen $\mathfrak{A}(z)=0$ und $\mathfrak{B}(z)=0$ durch dasselbe zugleich befriedigt werden müssten - ein Widerspruch. Da ebenso $\mathfrak{L}(z)$ keinen Theiler von $\mathfrak{A}(z)$ besitzt, ergibt sich aus

$$
\mathfrak{M}(z)=\mathfrak{A} \mathfrak{B}(z)=\mathfrak{K} \mathfrak{Q}(z)
$$

die Gleichheit von $\mathfrak{R}(z)$ mit $\mathfrak{H}(z)$ und $\mathfrak{L}(z)$ mit $\mathfrak{B}(z)$. Der Ausdruck $\mathfrak{\Omega}(z)$ ist von $x$-ten Grade und verschwindet genau für $x$ linear unabhängige Elemente aus $Q_{\mu}$, wodurch wir den

Satz II : Ist $\mathfrak{M}(z)=\mathfrak{A} \mathfrak{B}(z)$ d e r z u m G e bi e te $\Omega_{u}$ g ehörige charakteristische Ausdruck, so verschwindet der Theiler $\mathfrak{A}(z)$, wenn er mit $\mathfrak{B}(z)$ theilerfremd ist, genau für so viele Elemente aus $\Omega_{\mu}$, als sein Grad angib t

bewiesen haben.

Schreiben wir also

$$
\mathbb{E}^{\lambda-1}(z)=\mathbb{F}^{2}(z),
$$

so haben wir mit der Ergänzung $\mathbb{C}^{0}(z)=z$, $\mathbb{E}^{1}(z)=\mathbb{E}(z)$ für das Gebiet $\Omega_{\mu}$.den

Satz III: Entsprechend der Zerlegung des charakteristischen Ausdruckes $\mathfrak{F}(z)$ in

$$
\mathfrak{F}(z)=\mathfrak{F}_{1}^{\lambda_{1}} \mathfrak{F}_{2}^{\lambda_{2}} \ldots \mathbb{E}_{\sigma}^{\lambda_{\sigma}}(z)
$$

gibt es in $\Omega_{\mu}$ 


$$
\begin{aligned}
& \text { zu } \tilde{F}_{1}^{\lambda_{1}}(z)=0 \text { genau } \lambda_{1} \text { Elemente } \\
& " \tilde{E}_{2}^{\lambda_{2}}(z)=0 \quad, \quad \lambda_{2} \quad n \\
& " \dot{F}_{\sigma}^{\lambda_{\sigma}}(z)=0 \quad, \lambda_{\sigma} \quad, \quad,
\end{aligned}
$$

welche sämmtlich untereinander linear unabhängig sind und wegen $\lambda_{1}+\lambda_{2}+\ldots+\lambda_{\sigma}=n$ ein Fundamentalsystem in $\Omega_{\mu}$ bilden.

Wenn $E^{\lambda}(z)=0$ durch die $\lambda$ Elemente $x_{1}, x_{2}, \ldots, x_{\lambda}$ befriedigt wird, so besteht zwischen diesen und den Elementen $\Theta x_{1}, \Theta x_{2}, \ldots, \Theta x_{\lambda}$ eine lineare Substitution. Jedes dem Satze III entsprechende Fundamentalsystem wollen wir ein canonisches Fundamentalsystem und ebenso die dazugehörige Substitution $\mathrm{T} \Theta \mathrm{T}^{-1}$ in diesem Falle canonisch nennen. Es können zwar die Elemente der Theilgebiete noch weiter in Untergruppen eingetheilt werden, wobei die Matrix $\mathrm{T} \Theta \mathrm{T}^{-1}$ die sogenannte Normalform annimmt, denn es gibt nach dem Satze $I$ in jedem Theilgebiete sogar Elemente der Eigenschaft $\theta z-\omega z \doteq 0$. Auf diese Eintheilung kommt es jedoch hier nicht an, weshalb wir sie unterlassen; wir bemerken nur, dass wir durch die Substitution T, welche das canonische Fundamentalsystem mit dem ursprünglichen $z_{1}, z_{2}, \ldots, z_{n}$ verbindet, das Zerfallen der Substitution (Matrix) $\mathrm{T} \Theta \mathrm{T}^{-1}$ bewirkt haben.

4.

Nach diesen Entwickelungen wollen wir in $Q_{n}$ durch $p$ miteinander vertauschbare Substitutionen $\Theta_{1}, \Theta_{2}, \ldots, \Theta_{p}$ eine Zuordnung der Elemente $z u$ einander in dem im Abschnitt 1 angegebenen Sinne bestimmt annehmen. Die diesen Substitutionen entsprechenden charakteristischen Ausdrücke seien bezüglich $\mathfrak{F}_{1}(z), \mathfrak{F}_{2}(z), \ldots, \mathfrak{F}_{p}(z)$.

Wie wir bereits bewiesen haben, gibt es zu einem $\lambda$-fachen Lineartheiler $\mathfrak{F}_{1}^{\lambda}(z)$ von $\mathfrak{F}_{1}(z)$ genau $\lambda$ linear unabhängige Elemente, etwa

$$
z_{1}, z_{2}, \ldots, z_{*}
$$

für welche $\varepsilon_{1}^{\lambda}(z)=0$ wird. Aus (7) folgt wegen der Vertauschbarkeit der Substitutionen $\Theta_{1}$ und $\Theta_{2}$ die Gleichung $\left(\dot{E}_{1}^{\lambda} \Theta_{2} z=\Theta_{2} \dot{E}_{1}^{\lambda}(z)\right.$, woraus man ersieht, dass auch die Elemente

$$
\Theta_{2} z_{1}, \Theta_{2} z_{2}, \ldots, \Theta_{2} z_{2}
$$

der Gleichung $\mathbb{E}_{1}^{\lambda}(z)=0$ genügen, also durch $z_{1}, z_{2}, \ldots, z_{\lambda}$ darstellbar sind. Es sei diese Darstellung

$$
\left(\Theta_{2} z_{1}, \Theta_{2} z_{2}, \ldots, \Theta_{2} z_{\lambda}\right)=A\left(z_{1}, z_{2}, \ldots, z_{\lambda}\right)
$$


Man sieht also, dass die Substitutionen $\mathrm{T} \Theta_{1} \mathrm{~T}^{-1}$ und $\mathrm{T} \Theta_{2} \mathrm{~T}^{-1}$ gleichzeitig in derselben Weise zerfallen. Ausdruck

Aus der Substitution $A$ ergibt sich nach Abschnitt 2 ein

$$
\Re_{2}(z)=\Theta_{2}^{2} z+v_{1} \theta_{2}^{\lambda-1} z+\cdots+v_{\lambda-1} \Theta_{2} z+v_{\lambda} z
$$

der für sämmtliche Elemente $z_{1}, z_{2}, \ldots, z_{\lambda}$ verschwindet. $\Re_{2}(z)$ ist offenbar ein Theiler von $\mathfrak{F}_{2}(z)$. Ein $x$-facher Lineartheiler $\mathbb{F}_{2}^{*}(z)$ von $\mathfrak{R}_{2}(z)$ verschwindet nach dem Satze II genau für $x$ linear unabhängige Elemente, etwa

$$
z_{1}, z_{2}, \ldots, z_{x}
$$

Da nun aber nach (6) wieder $\mathscr{E}_{2}^{*} \Theta_{1} z=\Theta_{1} \tilde{\varepsilon}_{2}^{*}(z)$ folgt, so ver schwindet $\mathfrak{E}_{2}^{*}(z)$ auch für die Elemente

$$
\Theta_{1} z_{1}, \Theta_{1} z_{2}, \ldots, \Theta_{1} z_{x^{\prime}}
$$

Aus der Substitntion, welche demnach $z$ wischen $\theta_{1} z_{1}, \theta_{1} z_{2}, \ldots, \theta_{1} z_{x}$ und $z_{1}, z_{2}, \ldots, z_{x}$ bestehen muss, ergibt sich ein charakteristischer Ausdruck $x$-ten Grades, welcher als Theiler von $E_{1}^{\lambda}(z)$ nur $\mathfrak{F}_{1}^{\alpha}(z)$ sein kann. Die Elemente

$$
z_{1}, z_{2}, \ldots, z_{*}
$$

befriedigen folglich zugleich die beiden Gleichungen

$$
\xi_{1}^{*}(z)=0, \dot{E}_{2}^{*}(z)=0 .
$$

Ebenso können wir schließen, dass die Elemente

$$
\Theta_{3} z_{1}, \theta_{3} z_{2}, \ldots, \theta_{3} z_{x}
$$

diesen beiden Gleichungen genügen, also durch $z_{1}, z_{2}, \ldots, z_{x}$ linear darstellbar sind. Hebt man aus der sich ergebenden charakteristischen Function wieder einen Lineartheiler $\mathbb{E}_{3}(z)$ heraus und bezeichnet mit $\nu$ seine Vielfachheit, so gibt es genau $\nu$ linear unabhängige Elemente, welche $\mathbb{F}_{3}^{y}(z)=0$ entsprechen. Diese $v$ Elemente genügen also zugleich den drei Gleichungen

$$
\mathfrak{E}_{1}^{\prime}(z)=0, \mathbb{E}_{2}^{\nu}(z)=0, \mathfrak{E}_{3}^{\prime}(z)=0 \text {. }
$$

Auf gleiche Weise weiter schließend bekommen wir den

Satz IV. Es lässt sich im Gebiete $\Omega_{n}$ unter der Voraussetzung der Vertauschbarkeit der Substitutionen $\Theta_{1}, \Theta_{2}, \ldots, \Theta_{p}$ ein System von $n$ linear unabhängigen Elementen bestimmen, welche in derartige Gruppen zerfallen, dass sämmtliche Elemente einer solchen $m$-gliedrigen Gruppedie $p$ Gleichungen 


$$
\mathfrak{S}_{1}^{m}(z)=0, \mathbb{E}_{2}^{m}(z)=0, \ldots, \mathfrak{E}_{p}^{m}(z)=0
$$

zugleich befriedigen. Dabei ist

$$
\dot{\varepsilon}_{i}^{m}(z)=\Theta_{i}^{m} z-\left(\begin{array}{c}
m \\
1
\end{array}\right) \omega_{i} \Theta_{i}^{m-1} z+\left(\begin{array}{c}
m \\
2
\end{array}\right) \omega_{i}^{2} \theta_{i}^{m-2} z-\cdots+\left(-\omega_{i}\right)^{m} z
$$

und $\omega_{i}$ ist eine Wurzelderausder Substitution $\theta_{i}$ sich ergebenden charakteristischen Gleichung.

Durch dasselbe Verfahren, das wir hier beobachtet haben, läßt sich mit Leichtigkeit nachweisen, dass es in jeder Gruppe auch in diesem Falle mindestens ein Element gibt, für welches die $p$ Gleichungen

$$
\theta_{1} z=\omega_{1} z, \quad \theta_{2} z=\omega_{2} z, \ldots, \quad \Theta_{p} z=\omega_{p} z
$$

bestehen. Ein den Bedingungen des Satzes IV entsprechendes Fundamentalsystem in $\Omega_{n}$ hat die Eigenschaft, dass es gleichzeitig bezüglich aller Substitutionen canonisch ist.

Die Zahlen $1,2,3, \ldots, n$ lassen sich demnach in derartige Gruppen eintheilen, dass jede dieser Zahlen in einer und nur in einer Gruppe vorkommt, und, dass in allen Matricen $\mathrm{T} \theta_{1} \mathrm{~T}^{-1}$, $\mathrm{T}_{2} \mathrm{~T}^{-1}, \ldots, \mathrm{T} \Theta_{p} \mathrm{~T}^{-1}$ sämmtliche Coefficienten $a_{i k}$ verschwinden, wenn nicht $i$ und $k$ in dieselbe Gruppe fallen. Wir sagen, dass die Matricen $\Theta_{1}, \Theta_{2}, \ldots, \theta_{p}$ bei der Transformation dureh $\mathrm{T}$ gleichzeitig und zwar alle in derselben Weise zerfallen. Dem Satze IV können wir dann auch die Fassung geben:

$\mathrm{Zu}$ einer beliebigen Anzahl unter einander vertauschbaren linearen Substitutionen (Matricen) $\Theta_{1}, \Theta_{2}, \ldots, \Theta_{p}$ lässtsich stets eine derartige Substitution T auffinden, dass die ähnlichen Substitutionen $\mathrm{T}_{1} \mathrm{~T}^{-1}$, $\mathrm{T} \Theta_{2} \mathrm{~T}^{-1}, \ldots, \mathrm{T} \Theta_{p} \mathrm{~T}^{-1}$ in derselben $\mathrm{W}$ eise und zwarderart zerfallen, dass die charakteristischen Gleichungen einzelner Substitutionen, in welcheirgend e in $\mathrm{T}_{i} \mathrm{~T}^{-1}$ gespalten wurde, nur je eine, wenn auch vielleicht vielfache Wurzel besitzen.

\section{5.}

In Anwendung des erhaltenen Resultates auf lineare Differentialgleichungen lassen wir $z_{1}, z_{2}, \ldots, z_{n}$ ein Fundamentalsystem derselben bedeuten und wollen entsprechend mit $\Theta_{i} z_{1}, \Theta_{i} z_{2}, \ldots, \Theta_{i} z_{n}$ die Fortsetzungen seiner Elemente auf einem Wege $W_{i}$, der die Coefficienten der Differentialgleichung in die ursprünglichen Werte zurückführt und dem die Fundamentalsubstitution $\Theta_{i}$ in den $z_{1}, z_{2}, \ldots, z_{n}$ und ihren Fortsetzungen entspricht, bezeichnen. Die durch die Gleichungen (2) definierte Zuordnung der Elemente zu einander entspricht also 
den Fortsetzungen derselben. $\mathfrak{F}_{i}=0$ ist die zum Forsetzungswege $W_{i}$ gehörigen Fundamentalgleichung.

Besteht die Monodromiegruppe der Differentialgleichung in irgend einer Riemann'schen Fläche, die durch eine Anzahl Querschnitte einfach zusammenhängend gemacht wurde, aus lauter vertauschbaren Substitutionen, so ist die Differentialgleichung reductibel. Um dies einzusehen, sei

$$
x_{1}, x_{2}, \ldots, x_{m}
$$

irgend eine den Bedingungen des Satzes IV entsprechende Gruppe von particulären Integralen, welche die $p$ Gleichungen

$$
\mathfrak{E}_{1}^{m}(z)=0, \mathfrak{E}_{2}^{m}(z)=0, \ldots, \mathscr{E}_{p}^{m}(z)=0
$$

befriedigen, wobei $\mathbb{E}_{1}(z), \mathbb{E}_{2}(z), \ldots, \mathbb{E}_{p}(z)$ Lineartheiler der aus den Fundamentalsubstitutionen $\Theta_{1}, \Theta_{2}, \ldots \Theta_{p}$ sich ergebenden charakteristischen Ausdrücke sind, $\mathbb{E}_{i}^{m}$ also in der zur Substitution $\theta_{i}$ gehörigen Fundamentalgleichung $\mathfrak{F}_{i}=0$ "linkerseits" als Theiler enthalten ist. $\mathrm{Da}$ jedes particuläre Integral, welches diesen sämmtlichen Gleichungen entspricht, nach dem bewiesenen durch $x_{1}, x_{2}, \ldots, x_{m}$ linear darstellbar ist, so gilt dies auch von

$$
\Theta_{i} x_{1}, \Theta_{i} x_{2}, \ldots, \Theta_{i} x_{m},
$$

weil aber der lineare Differentialausdruck

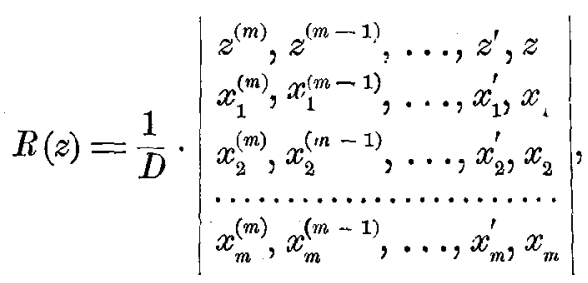

wo mit $D$ die nicht identisch verschwindende Subdeterminante von $z^{(m)}$ und mit $x^{(\lambda)}$ die $\lambda$-te Derivierte von $x$ bezeichnet wurde, bei allen linearen Substitutionen in $x_{1}, x_{2}, \ldots, x_{m}$ invariant bleibt, so genügen sämmtliche particulären Integrale sammt allen ihren Fortsetzungen der linearen Differentialgleichung

$$
R(z)=0,
$$

welche eindeutige Coefficienten besitzt. Die Monodromiegruppe dieser Differentialgleichung besteht ebenfalls aus lauter vertauschbaren Substitutionen und die Fundamentalgleichungen haben sämmtlich nur je eine, wenn auch mehrfache Wurzel.

Nach unseren Entwickelungen besitzt die Differentialgleichung mindestens ein particuläres Integral der Eigenschaft

$$
\Theta_{1} x=\omega_{1} x, \quad \Theta_{2} x=\omega_{2} x, \ldots \quad \Theta_{p} x \doteq \omega_{p} x
$$


dessen logarithmische Ableitung demnach eindeutig ist. Das Integral $x$ genügt also einer linearen Differentialgleichung erster Ordnung

$$
E(z)=0
$$

mit eindeutigen Coefficienten. Setzt man nun

$$
R(z)=R_{1} E(z),
$$

so hat die Differentialgleichung $(m-1)$-ter Ordnung

$$
R_{1}(z)=0
$$

wegen

$$
\Theta_{i_{1}}^{\alpha_{1}} \Theta_{i_{2}}^{\alpha_{z}} F(z)=E\left(\Theta_{i_{1}}^{\alpha_{1}} \Theta_{i_{2}}^{\alpha_{2}} z\right)=\Theta_{i_{2}}^{\alpha_{2}} \theta_{i_{3}}^{\alpha_{1}} E(z)
$$

ebenfalls eine vertauschbare Monodromiegruppe und eindeutige Coefficienten, wodurch nachgewiesen ist, dass die Integration einer linearen Differentialgleichung mit vertauschbarer Monodromiegruppe auf eire Kette von linearen Differentialgleichungen erster Ordnung mit eindeutigen Coefficienten zurückführbar ist, also durch Quadrataren geleistet werden kann. Aber selbst die analytische Form der Integrale lässt sich, wie nach $F u c h s$ aus der canonischen Fundamentalsubstitution für die Umgebung eines singulären Punktes, hier zugleich für die Riemann'sche Fläche angeben. Der vielbehandelte Fall von Differentialgleichung mit doppeltperiodischen Coefficienten und eindeutigen Integralen ordnet sich hier als ein einfachster Specialfall ein. 